\title{
IMPACTO DE LAS CONDICIONES ORALES SOBRE LA CALIDAD DE VIDA EN ESCOLARES DE 11 Y 12 AÑOS DE EDAD DE ZAPALLAL-PUENTE PIEDRA
}

\section{ORAL CONDITIONS IMPACT ON THE QUALITY OF LIFE IN SCHOOL CHILDREN OF 11 AND 12 YEARS OF AGE OF ZAPALLAL-PUENTE PIEDRA}

\author{
Sánchez Borjas Pablo, ${ }^{1 *}$ Herrera Armijos Dayana. ${ }^{2}$ \\ ${ }^{1}$ Magíster en Estomatología.Profesor del Departamento Académico de Odontología Social Universidad Peruana Cayetano \\ Heredia. Perú. \\ 2 Odontóloga Rural del Ministerio de Salud Pública del Ecuador. Ecuador. \\ *pablo.sanchez@upch.pe
}

\section{Resumen}

OBJETIVO. El propósito de este estudio fue evaluar el impacto de las condiciones orales sobre la calidad de vida a escolares de 11 y 12 años de Zapallal - Puente Piedra. MATERIALES Y MÉTODOS. Se seleccionaron 4 de las 7 instituciones educativas públicas dentro del área de influencia del Centro Materno-Infantil de Zapallal (Puente Piedra), 805 de los 903 escolares de 11 y 12 años de edad, que asistían a estas cuatro instituciones, aceptaron participar del estudio (tasa de norespuesta de $10.9 \%$ ). Se realizaron entrevistas estructuradas cara a cara empleando la versión peruana del Child-OIDP. RESULTADOS. La prevalencia del impacto de las condiciones orales sobre la calidad de vida fue de $82.0 \%$ mientras que para la severidad se encontró un puntaje promedio de $7.77 \pm 8.64 \%$. Entre los 660 niños que reportaron impactos, la mayoría de ellos $(82.7 \%)$ presentaron poca a muy poca intensidad, mientras que el número promedio de actividades diarias impactadas fue de $2.23 \pm 1.70$, siendo comer, limpiarse la boca y sonreír las actividades diarias más frecuentemente impactadas. CONCLUSIÓN. El dolor de muela fue la causa principal de los impactos sobre todas las actividades diarias con excepción de sonreír, cuya causa principal fue la alteración en la posición de los dientes. Los presentes resultados resaltan la utilidad de evaluar las necesidades auto-percibidas, como complemento a los indicadores clínicos habitualmente empleados al determinar las necesidades de tratamiento buco-dental, así como, para planificar servicios para el cuidado de la salud oral en el ámbito comunitario.

Palabras clave: Calidad de vida, salud oral, niños.

\begin{abstract}
OBJECTIVE The purpose of this study was to evaluate the impact of oral conditions on the quality of life of schoolchildren aged 11 and 12 years of Zapallal - Puente Piedra. MATERIALS AND METHODS. 4 of the 7 public educational institutions were selected within the area of influence of the Maternal-Infant Center of Zapallal (Puente Piedra), 805 of the 903 schoolchildren of 11 and 12 years of age, who attended these four institutions, agreed to participate in the study (non-response rate of $10.9 \%$ ). Structured face to -face interviews were conducted using the Peruvian version of Child-OIDP. RESULTS. The prevalence of the impact of the oral conditions on the quality of life was $82.0 \%$ while for the severity an average score of 7.77 underline $+8.64 \%$ was found. Among the 660 children who reported impacts, most of them (82.7\%) showed little to very little intensity, while the average number of daily activities impacted was $2.23+$ line 1.70, being eating, cleaning the mouth and Smile the daily activities most frequently impacted. CONCLUSION. Toothache was the main cause of the impact on all daily activities except for smiling, whose main cause was the alteration in the position of the teeth. The present results highlight the usefulness of assessing self-perceived needs, as a complement to the clinical indicators commonly used to determine the needs of oral-dental treatment, as well as to plan services for oral health care in the community.
\end{abstract}

Key words: Quality of life, oral health, children . 


\begin{tabular}{ccccc}
\hline \multirow{2}{*}{ Condiciones orales } & \multicolumn{2}{c}{ Presente } & \multicolumn{2}{c}{ Ausente } \\
\cline { 2 - 5 } & $\mathbf{N}$ & $\mathbf{\%}$ & $\mathbf{n}$ & $\mathbf{\%}$ \\
\hline Dolor de muela & 345 & 42.9 & 460 & 57.1 \\
Diente sensible & 269 & 33.4 & 536 & 66.6 \\
Diente cariado & 220 & 27.3 & 585 & 72.7 \\
Caída de un diente de leche & 211 & 26.2 & 594 & 73.8 \\
Dientes separados & 130 & 16.1 & 675 & 83.9 \\
Fractura de diente permanente & 40 & 5.0 & 765 & 95.0 \\
Color de los dientes & 175 & 21.7 & 630 & 78.3 \\
Forma o tamaño de los dientes & 113 & 14.0 & 692 & 86.0 \\
Posición de los dientes & 227 & 28.2 & 578 & 71.8 \\
Sangrado de encías & 247 & 30.7 & 558 & 69.3 \\
Inflamación de las encías & 140 & 17.4 & 665 & 82.6 \\
Sarro o cálculo dental & 118 & 14.7 & 687 & 85.3 \\
Llagas o heridas en la boca & 132 & 16.4 & 673 & 83.6 \\
Mal aliento & 192 & 23.9 & 613 & 76.1 \\
Deformación de la boca o cara & 13 & 1.6 & 792 & 98.4 \\
Erupción de diente permanente & 17 & 2.1 & 788 & 97.9 \\
Pérdida de diente permanente & 65 & 8.1 & 740 & 91.9 \\
Otros & 6 & 0.7 & 799 & 99.3 \\
\hline
\end{tabular}

Tabla 1. Frecuencia de condiciones orales auto-percibidas como problemas por los escolares de 11 y 12 años de Zapallal (Puente Piedra, 2006)

\begin{tabular}{cccccc}
\hline \multirow{2}{*}{ Condiciones orales } & \multicolumn{2}{c}{$\begin{array}{c}\text { Mujeres } \\
(\mathbf{n = 4 1 2})\end{array}$} & \multicolumn{2}{c}{$\begin{array}{c}\text { Varones } \\
(\mathbf{n}=\mathbf{3 9 3})\end{array}$} & \multirow{2}{*}{ valor } \\
\cline { 2 - 5 } & $\mathbf{N}$ & $\mathbf{\%}$ & $\mathbf{n}$ & $\mathbf{\%}$ & $\mathbf{p}$ \\
\hline Dolor de muela & 167 & 40.5 & 178 & 45.3 & 0.173 \\
Diente sensible & 152 & 36.9 & 117 & 29.8 & 0.032 \\
Diente cariado & 106 & 25.7 & 114 & 29.0 & 0.297 \\
Caída de un diente de leche & 110 & 26.7 & 101 & 25.7 & 0.747 \\
Dientes separados & 53 & 12.9 & 77 & 19.6 & 0.010 \\
Fractura de diente permanente & 10 & 2.4 & 30 & 7.6 & 0.001 \\
Color de los dientes & 95 & 23.1 & 80 & 20.4 & 0.353 \\
Forma o tamaño de los dientes & 43 & 10.4 & 70 & 17.8 & 0.003 \\
Posición de los dientes & 117 & 28.4 & 110 & 28.0 & 0.898 \\
Sangrado de encías & 120 & 29.1 & 127 & 32.3 & 0.327 \\
Inflamación de las encías & 61 & 14.8 & 79 & 20.1 & 0.048 \\
Sarro o cálculo dental & 55 & 13.3 & 63 & 16.0 & 0.282 \\
Llagas o heridas en la boca & 65 & 15.8 & 67 & 17.0 & 0.626 \\
Mal aliento & 81 & 19.7 & 111 & 28.2 & 0.004 \\
Deformación de la boca o cara & 7 & 1.7 & 6 & 1.5 & 0.846 \\
Erupción de diente permanente & 6 & 1.5 & 11 & 2.8 & 0.185 \\
Pérdida de diente permanente & 17 & 4.1 & 48 & 12.2 & $<0.001$ \\
Otros & 4 & 1.0 & 2 & 0.5 & 0.446 \\
\hline Se empleó la prueba Chi-cuadrado & & & &
\end{tabular}

Tabla 2. Frecuencia de condiciones orales auto-percibidas como problemas por los escolares de 11 y 12 años de Zapallal según sexo (Puente Piedra, 2006)

\section{INTRODUCCIÓN}

En salud pública dental, la planificación de intervenciones comunitarias requiere contar con información sobre la necesidad de tratamiento de las condiciones que afectan la salud oral de la población. ${ }^{1}$ Con esta finalidad, múltiples escalas o índices han sido propuestos; sin embargo, la mayoría de ellos se basan en mediciones clínicas objetivas determinadas por el profesional (salud física), descuidando desde todo punto de vista los componentes mental y social de las condiciones orales. ${ }^{2,3}$ Medir la salud significa que los índices clínicos que valoran la presencia y/o severidad de las condiciones patológicas necesitan ser complementados por mediciones que reflejen los aspectos emocionales y sociales de la salud. ${ }^{4}$ Tales medidas, basadas sobre estados emocionales auto-percibidos y funcionamiento psicológico como por ejemplo la capacidad de las personas para llevar a cabo los roles deseados, ${ }^{5} \mathrm{o}$ de la satisfacción de las personas al realizar importantes habilidades de la vida diaria como el masticar, hablar o dormir, ${ }^{6,7}$ permitirán documentar las consecuencias personales inmediatas y sociales más amplias de las enfermedades. La creciente preocupación acerca de los conceptos multidimensionales de la salud oral así como la inadecuación del enfoque normativo (uso de indicadores clínicos) han guiado al desarrollo de marcos teóricos e instrumentos de medición de la Calidad de Vida Relacionada a la Salud Oral (CVRSO) representando las consecuencias físicas, psicológicas, funcionales y sociales de las condiciones orales. ${ }^{8}$ Del mismo modo, nuevos instrumentos de medición, comúnmente denominados indicadores socio-dentales, han sido desarrollados para complementar los indicadores clínicos convencionalmente empleados en la práctica dental, así como para investigar y proveer una mayor información basal que permita la evaluación de la salud oral a escala individual y colectiva. De acuerdo con tales modificaciones, existen tres diferentes niveles de consecuencias sobre la vida diaria que provocan las condiciones orales (Figura 1).

Desde entonces, múltiples equipos de investigadores, trabajando independientemente, ${ }^{9-17}$ han desarrollado instrumentos para la evaluación de los impactos que las condiciones orales provocan sobre la vida diaria de las personas, así como para evaluar el beneficio del cuidado brindado cuando ellos son empleados para complementar las medidas tradicionales basadas en enfermedad. La mayoría de estudios sobre CVRSO han sido llevados a cabo en poblaciones de adultos o adultos mayores. ${ }^{18-22}$ Pocos estudios han sido conducidos en poblaciones más jóvenes como niños o adolescentes, debido a que hasta hace algunos años ninguna medida de CVRSO había sido diseñada para su uso específico en este tipo de poblaciones. En el 2004, se reportó el desarrollo y validación del Child-OIDP en Tailandia, como una versión que permite evaluar los impactos de las condiciones orales sobre la vida diaria de niños entre 11 y 12 años de edad. Las actividades evaluadas son comer, hablar, limpiarse la boca, estudiar, dormir, sonreír, contactarse con otras personas y mantener un buen estado emocional. El Child-OIDP ha demostrado poseer muchas de las características ideales en un índice epidemiológico reportadas en la literatura. ${ }^{23-27}$ Es un instrumento corto, rápido y entretenido así como también posee excelentes propiedades psicométricas (validez y confiabilidad). Recientemente, dicho instrumento ha sido adaptado y validado para su uso entre niños de Francia ${ }^{28}$ y del Reino Unido. ${ }^{29}$ Asimismo, una versión peruana del instrumento, realizada en una población de niños de 11 y 12 años del distrito de Puente Piedra, ha sido recientemente traducida, adaptada y validada, encontrándose en fase de publicación 


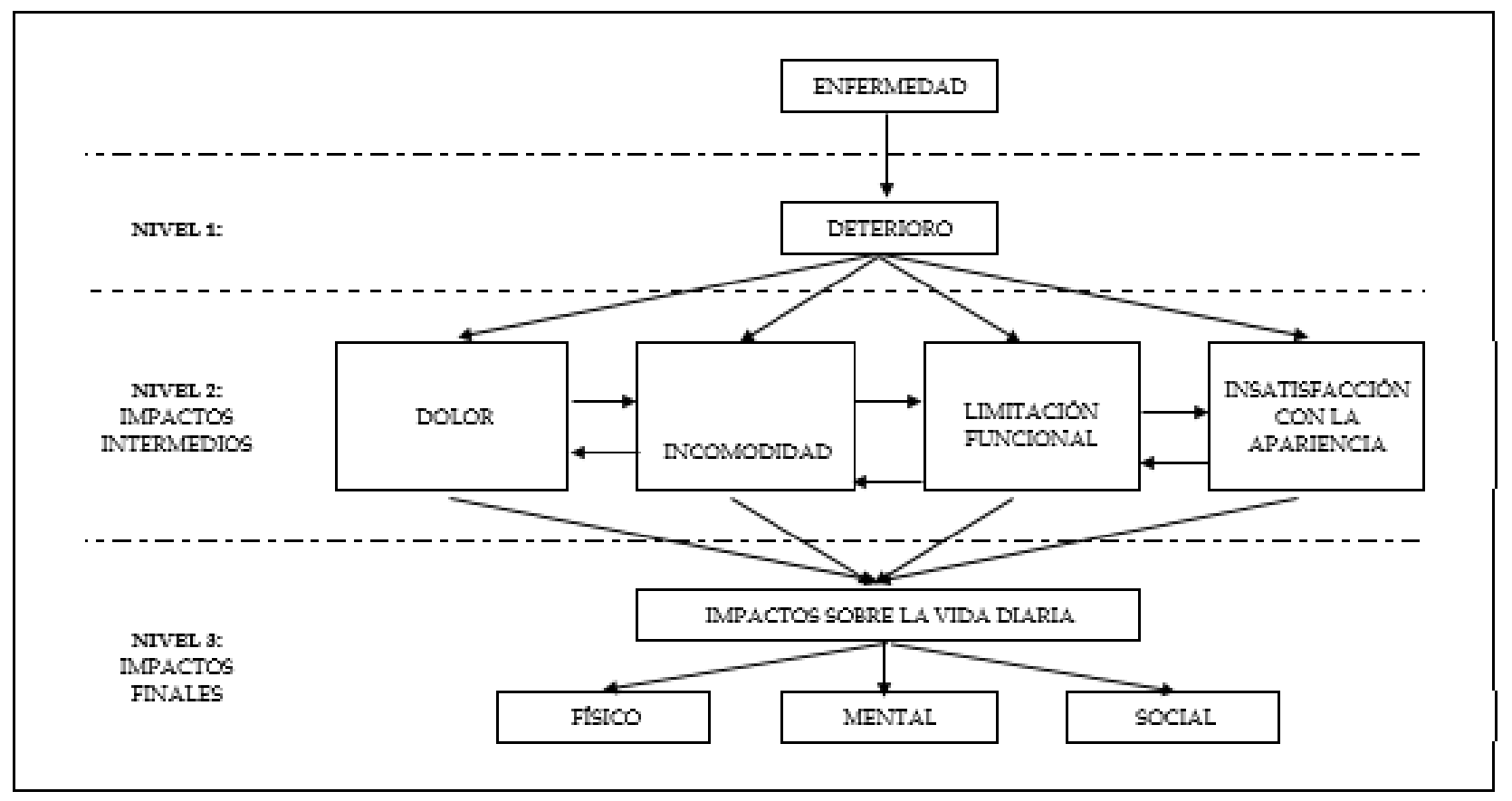

Fig. 1. Modelo de Salud Oral con las consecuencias de los impactos orales ${ }^{12}$

en la literatura internacional. ${ }^{30}$ Todos estos estudios han sido llevados a cabo bajo asesoría del equipo de investigadores que diseñó y desarrolló la versión original del Child-OIDP (Universidad de Londres en el Reino Unido). Mediante el uso del Child-OIDP se ha podido corroborar que las condiciones orales afectan la calidad de vida de los niños, a través de la dificultad para realizar determinadas actividades de la vida diaria. A pesar de ello, existen muy pocos estudios sobre dicho impacto en poblaciones de niños y adolescentes fuera de Norteamérica y Europa, ${ }^{31-33}$ especialmente en Latinoamérica. ${ }^{34-36}$ Por lo anteriormente expuesto, la utilidad del presente estudio es ampliar el conocimiento que se tiene acerca de estos problemas de salud tanto a escala individual como colectiva, lo cual servirá para el desarrollo y comprobación de modelos de atención en salud oral como también para identificar posibles determinantes de la salud oral infantil y expandir los métodos actuales (enfoque normativo) empleados para la evaluación y planificación de programas o servicios odontológicos, asistiendo en la toma de decisiones acerca de quienes deben recibir atención prioritaria para la resolución de sus problemas de salud oral, asegurando una mejor asignación de los escasos recursos existentes en el sector. Finalmente, el propósito del presente estudio es evaluar el impacto de las condiciones orales sobre la calidad de vida en niños de 11 y 12 años de edad provenientes de instituciones educativas de Zapallal en Puente Piedra.

\section{MATERIALES Y MÉTODOS}

El diseño del presente estudio fue observacional y descriptivo de corte transversal, los datos fueron recogidos mediante entrevistas estructuradas cara a cara, con ayuda de un cuestionario de dos secciones. La primera sección permitió determinar la institución educativa y año de estudios de cada entrevistado. La segunda sección estuvo conformada por la versión peruana del Child-OIDP, la cual había sido previamente adaptada y validada para recoger información sobre el impacto de las condiciones orales sobre la vida diaria. la población estuvo conformada por 1519 niños de 11 y 12 años de edad (nacidos en 1995 y 1994 respectivamente), que asistían en el año 2006 a las siete instituciones educativas públicas de nivel primario y secundario, localizadas dentro del área de influencia del Centro Materno-Infantil de Zapallal. Para la selección del tamaño de la muestra fue calculado mediante una fórmula, que se conformó por 805 niños de 11 y 12 años de edad, quienes dieron su asentimiento escrito y cuyos padres firmaron un consentimiento informado aceptando su participación en el estudio. El puntaje total para el Child-OIDP se obtuvo con las respuestas brindadas por cada entrevistado, multiplicando los puntajes correspondientes a frecuencia y severidad $(0,1,2$ y 3 en cada uno) para cada una de las ocho actividades de la vida diaria evaluadas. De esta forma, el puntaje individual por actividad diaria variaba entre 0 y 9 puntos, mientras que el puntaje total lo hacía entre 0 y 72 puntos al sumar los ocho puntajes individuales. 


\begin{tabular}{lcccccc}
\hline \multicolumn{1}{c}{ Covariables } & $\mathbf{n}$ & Media & D.E. & $\begin{array}{c}\text { Valor } \\
\text { mínimo }\end{array}$ & $\begin{array}{c}\text { Valor } \\
\text { máximo }\end{array}$ & $\begin{array}{c}\text { Valor } \\
\mathbf{p}\end{array}$ \\
\hline $\begin{array}{l}\text { Sexo } \\
\text { Mujer }\end{array}$ & 412 & 3.08 & 2.26 & 0 & 12 & \\
$\quad \begin{array}{l}\text { Varón } \\
\text { Nivel educativo }\end{array}$ & 393 & 3.54 & 2.68 & 0 & 14 & \\
$\quad$ & & & & & 0.033 \\
Primaria & 431 & 3.51 & 2.53 & 0 & 14 & \\
Secundaria & 374 & 3.06 & 2.40 & 0 & 12 & \\
TOTAL & 805 & 3.30 & 2.48 & 0 & 14 & $-\ldots$ \\
\hline \multicolumn{2}{l}{$\begin{array}{l}\text { D.E. = Desviación Estándar } \\
\text { Se empleó la prueba U de Mann-Whitney }\end{array}$}
\end{tabular}

Tabla 3. Número promedio de condiciones orales auto-percibidas como problemas por los escolares de 11 y 12 años de Zapallal según covariables (Puente Piedra, 2006)

El puntaje total es presentado como porcentaje al sumar los ocho puntajes individuales, dividirlo entre 72 y multiplicarlo por 100, representando la severidad del impacto. Finalmente, para determinar la intensidad del impacto de las condiciones orales entre aquellos que reportaron impactos se empleó la clasificación sugerida por Gherunpong y et al. ${ }^{37} \mathrm{La}$ intensidad se refiere al impacto más severo sobre cualquiera de las ocho actividades diarias (puntaje individual más alto) mientras que la extensión se refiere al número de actividades diarias con impactos que afectan la vida diaria del niño (pudiendo variar entre 1 y 8 ). ${ }^{38}$

\section{RESULTADOS}

En el análisis de la frecuencia de las condiciones orales auto-percibidas como problemas, el dolor de muela y diente sensible fueron reportadas por más de un tercio de la muestra (Tabla 1).

Según el sexo se encontró que los varones reportaron con mayor frecuencia mal aliento, inflamación de encías, dientes separados, pérdida de un diente permanente, alteraciones en la forma o tamaño de los dientes y fractura de un diente permanente mientras que las mujeres reportaron con mayor frecuencia el diente sensible (Tabla 2), siendo estas diferencias estadísticamente significativas ( $\mathrm{p}<0.048$ ).

El número promedio de condiciones orales autopercibidas como problemas por los niños entrevistados fue en promedio de $3.30 \pm 2.48$, encontrándose también que 65 niños $(8.1 \%)$ no reportaron problema alguno. Al comparar el número de condiciones orales auto-percibidas como problemas según covariables (Tabla 3), se encontró mayor número en varones que en mujeres (3.54 contra 3.08), así como también en escolares de primaria que de secundaria (3.51 contra 3.06), siendo ambas diferencias estadísticamente significativas ( $\mathrm{p}=0.033$ y 0.009 respectivamente).

Al evaluar la prevalencia del impacto de las condiciones orales sobre la calidad de vida (Tabla 4), se encontró que el $82.0 \%$ de los escolares reportaron dificultades para realizar sus actividades diarias (Child-OIDP $>0$ ), siendo comer y estudiar las actividades con mayor y menor prevalencia de impactos respectivamente $(48.0 \%$ y $10.9 \%)$. Al evaluar la severidad del impacto de las condiciones orales sobre la calidad de vida (Tabla 5), se encontró un puntaje promedio de $7.77 \pm 8.64 \%$ en el Child-OIDP, siendo comer y estudiar las actividades diarias con mayor y menor severidad respectivamente $(1.04 \pm 0.56$ y $0.30 \pm 1.13$ puntos $)$. Al evaluar la intensidad del impacto de las condiciones orales sobre la calidad de vida entre los 660 escolares que reportaron impactos, se encontró que la mayoría de ellos (82.7\%) tuvo impactos de poca o muy poca intensidad, lo cual ocurrió también para los impactos sobre cada actividad diaria (Tabla 6). Al evaluar la extensión del impacto de las condiciones orales sobre la calidad de vida entre los 660 escolares que reportaron impactos, se encontró un promedio de $2.23 \pm 1.70$ para el número de actividades diarias con impactos, y que sólo el $12.3 \%$ de estos escolares presentaron impactos sobre 5 ó más actividades diarias. Al evaluar las condiciones orales percibidas como causas del impacto sobre la calidad de vida se encontró que el dolor de muela fue la causa principal de los 


\begin{tabular}{ccccc}
\hline $\begin{array}{c}\text { Actividades } \\
\text { diarias }\end{array}$ & \multicolumn{2}{c}{$\begin{array}{c}\text { Con impacto } \\
\text { (Child-OIDP }>\mathbf{0})\end{array}$} & \multicolumn{2}{c}{$\begin{array}{c}\text { Sin impacto } \\
(\mathbf{C h i l d - O I D P}=\mathbf{0})\end{array}$} \\
\cline { 2 - 5 } & $\mathbf{N}$ & $\mathbf{\%}$ & $\mathbf{n}$ & $\mathbf{\%}$ \\
\hline Comer & 386 & 48.0 & 419 & 52.0 \\
Hablar & 128 & 15.9 & 677 & 84.1 \\
Limpiarse la boca & 307 & 38.1 & 498 & 61.9 \\
Dormir & 195 & 24.2 & 610 & 75.8 \\
Emoción & 275 & 34.2 & 530 & 65.8 \\
Sonreir & 288 & 35.8 & 517 & 64.2 \\
Estudiar & 88 & 10.9 & 717 & 89.1 \\
Socializar & 125 & 15.5 & 680 & 84.5 \\
TOTAL & 660 & 82.0 & 145 & 18.0 \\
\hline
\end{tabular}

Tabla 4. Prevalencia del impacto de las condiciones orales sobre la calidad de vida en escolares de 11 y 12 años de Zapallal (Puente Piedra, 2006)

impactos sobre todas las actividades diarias con excepción de sonreír, cuya causa principal fue la alteración en la posición de los dientes (Gráfico 1). Socializar y sonreír fueron las actividades diarias con mayores diferencias según el sexo en relación con las condiciones orales causantes de los impactos (Gráfico 2), mientras que socializar fue la actividad diaria donde las condiciones orales percibidas como causas fueron diferentes según nivel educativo (Gráfico 3).

\section{DISCUSIÓN}

Para el presente estudio se entrevistaron escolares de 11 y 12 años de edad que asistían a cuatro de las ocho instituciones educativas públicas de nivel primario y secundario de Zapallal en Puente Piedra. En dicha comunidad, la carencia de servicios básicos (energía eléctrica y red de agua y desagüe) así como el pobre acceso a servicios de salud, recolección de basura y transporte público son las características socio-económicas predominantes. Aunque se utilizó un muestreo por conveniencia, seleccionando aquellas instituciones educativas donde el Departamento Académico de Odontología Social realiza labores de docencia en servicio, la participación de 805 de los 1519 (52.9\%) escolares en las siete instituciones educativas bajo la jurisdicción del Centro de Salud de la zona podría garantizar la extrapolación de los hallazgos a toda la población de estudio, debido también a la ausencia de informes previos sobre el tema de investigación. La entrevistas se realizaron a escolares entre 11 y 12 años de edad, debido a que éste es el rango de edad al cual está dirigida la versión original del Child-OIDP. ${ }^{39}$ Ha sido reportado que el uso de oraciones condicionales así como la capacidad de recordar eventos en el pasado, actividades requeridas durante las entrevistas realizadas, sólo llega a ser una práctica común hasta la edad de 11 y 12 años. Adicionalmente, éste es el rango de edad recomendado por la OMS para efectuar la vigilancia de las enfermedades orales así como para comparaciones internacionales. ${ }^{40}$ En respuesta al creciente reconocimiento de medir la calidad de vida como indicador del cuidado de la salud, diferentes indicadores que reconocen las consecuencias sociales y psicológicas de las condiciones orales han sido desarrollados para complementar los índices clínicos convencionales. ${ }^{24}$ En el presente estudio se empleó la versión peruana del Child-OIDP, la cual ha sido previamente traducida, adaptada y validada para su uso con niños peruanos. ${ }^{30}$ Una de las ventajas del Child-OIDP es que permite evaluar no sólo la prevalencia del impacto de las condiciones orales sobre la calidad de vida, sino también la severidad, intensidad y extensión de estos impactos, haciéndolo útil para su uso durante la evaluación de individuos y poblaciones. ${ }^{6}$ En relación con los resultados se encontró que la prevalencia del impacto de las condiciones orales sobre la calidad de vida fue muy alta, puesto que $82 \%$ de los niños entrevistados reportaron al menos un impacto afectan- 


\begin{tabular}{ccccc}
\hline $\begin{array}{c}\text { Actividades } \\
\text { diarias }\end{array}$ & $\begin{array}{c}\text { Media } \\
\mathbf{( \% )}\end{array}$ & $\begin{array}{c}\text { D.E. } \\
\mathbf{( \% )}\end{array}$ & $\begin{array}{c}\text { Valor } \\
\text { mínimo }\end{array}$ & $\begin{array}{c}\text { Valor } \\
\text { máximo }\end{array}$ \\
\hline Comer & 1.04 & 1.56 & 0 & 9 \\
Hablar & 0.37 & 1.08 & 0 & 9 \\
Limpiarse la boca & 0.93 & 1.61 & 0 & 9 \\
Dormir & 0.62 & 1.47 & 0 & 9 \\
Emoción & 0.85 & 1.62 & 0 & 9 \\
Sonreír & 1.06 & 1.92 & 0 & 9 \\
Estudiar & 0.30 & 1.13 & 0 & 9 \\
Socializar & 0.44 & 1.32 & 0 & 9 \\
TOTAL & 7.77 & 8.64 & 0 & 62.50 \\
\hline
\end{tabular}

D.E. = Desviación Estándar

Tabla 5. Severidad del impacto de las condiciones orales sobre la calidad de vida en escolares de 11 y 12 años de Zapallal (Puente Piedra, 2006)

\begin{tabular}{cccccccccc}
\hline & \multicolumn{10}{c}{ Actividades diarias } \\
\cline { 2 - 9 } Intensidad & $\begin{array}{c}\text { Comer } \\
\text { \% }\end{array}$ & $\begin{array}{c}\text { Hablar } \\
\text { \% }\end{array}$ & $\begin{array}{c}\text { Limpiarse } \\
\text { la boca } \\
\text { \% }\end{array}$ & $\begin{array}{c}\text { Dormir } \\
\text { \% }\end{array}$ & $\begin{array}{c}\text { Emoción } \\
\text { \% }\end{array}$ & $\begin{array}{c}\text { Sonreír } \\
\text { \% }\end{array}$ & $\begin{array}{c}\text { Estudiar } \\
\%\end{array}$ & $\begin{array}{c}\text { Socializar } \\
\%\end{array}$ & TOTAL \\
\hline Muy poca & 41.4 & 39.8 & 35.8 & 39.5 & 39.3 & 26.7 & 37.5 & 29.6 & 54.4 \\
Poca & 37.3 & 32.0 & 33.6 & 28.7 & 28.4 & 32.6 & 30.7 & 29.6 & 28.3 \\
Moderada & 13.5 & 18.0 & 19.2 & 17.4 & 19.3 & 21.9 & 14.8 & 26.4 & 15.5 \\
Severa & 5.7 & 8.6 & 9.1 & 10.8 & 10.5 & 13.2 & 10.2 & 8.8 & 1.5 \\
Muy severa & 2.1 & 1.6 & 2.3 & 3.6 & 2.5 & 5.6 & 6.8 & 5.6 & 0.3 \\
$(n)$ & $(386)$ & $(128)$ & $(307)$ & $(195)$ & $(275)$ & $(288)$ & $(88)$ & $(125)$ & $(660)$ \\
\hline
\end{tabular}

Tabla 6. Intensidad del impacto de las condiciones orales sobre la calidad de vida en escolares de 11 y 12 años de Zapallal (Puente Piedra, 2006)

do sus actividades diarias durante los últimos tres meses, lo cual es bastante similar a los valores reportados entre niños franceses $^{28}$ y tailandeses $(73 \%$ y $89.8 \%$ respectivamente). Estos resultados sugieren que el impacto de las condiciones orales suele ser muy común entre niños de 11 y 12 años de edad, y también mucho más frecuente que en otros grupos de edad según reportes de otros estudios. Así, al emplear el OIDP se encontró que la prevalencia de impactos fue bastante menor entre adolescentes de Brasil $(32.8 \%)^{19,20}$ y Uganda
$(62 \%),{ }^{33}$ adultos jóvenes de Tanzania $(51 \%),{ }^{24} \mathrm{y}$ adultos mayores de Tailandia $(52.8 \%),{ }^{18}$ Grecia $(39 \%)^{31}$ y Reino Unido $(12.3 \%)$. La alta prevalencia de impactos entre los niños entrevistados puede ser explicada por los altos niveles de caries dental existente en la población infantil peruana. En el Perú, el $84.0 \%$ de los niños presentan caries dental a los 12 años de edad, ${ }^{34}$ con un promedio de 3.1 dientes permanentes con experiencia de caries dental. ${ }^{35,36}$ Esto es también sustentado por el hecho que las dos principales condiciones orales 


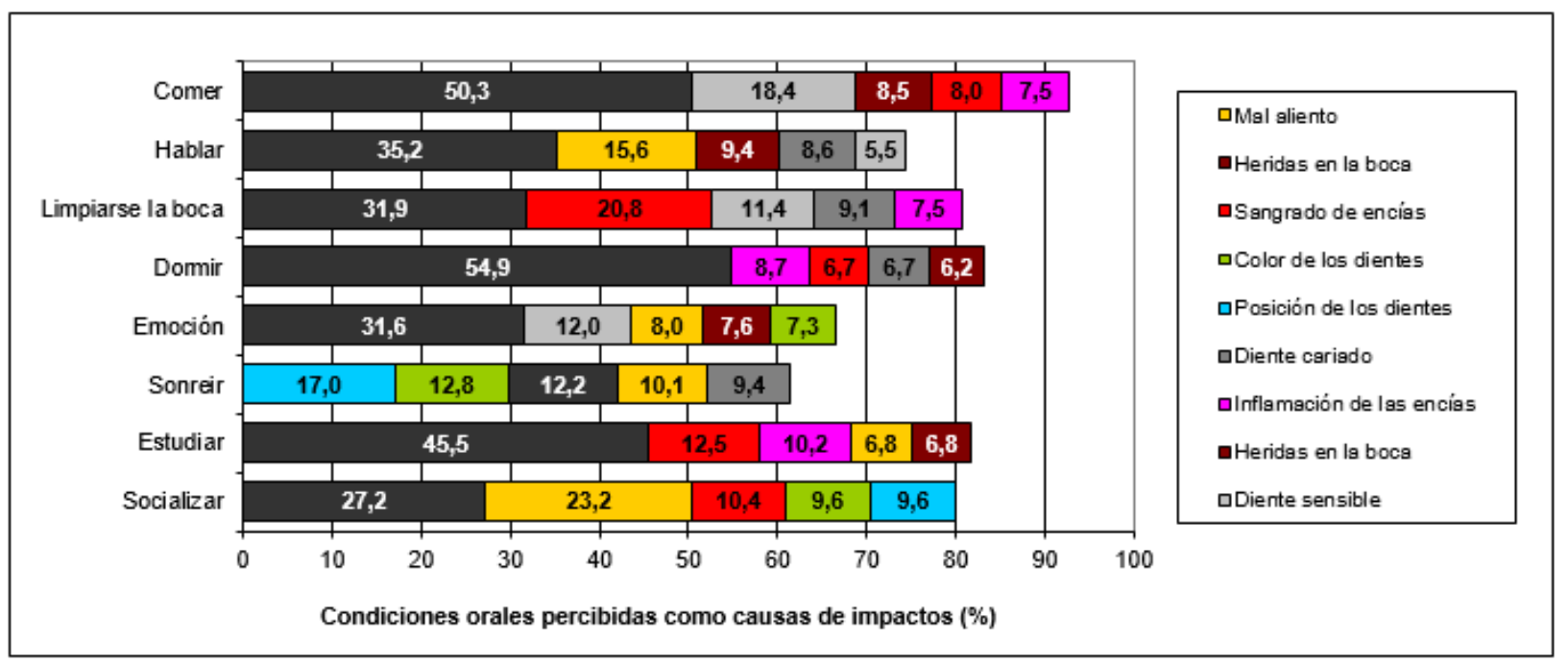

Grf. 1. Condiciones orales percibidas como causas del impacto sobre la calidad de vida en escolares de 11 y 12 años de Zapallal (Puente Piedra, 2006.)

auto-percibidas como problemas por los niños entrevistados fueron el dolor de muela y el diente sensible (Tabla 2), dos síntomas muy relacionados con los estadios avanzados de la enfermedad caries dental. Adicionalmente se encontró que la mayoría de los impactos estuvieron relacionados con dificultades para comer y limpiarse la boca, confirmando resultados de estudios previos. ${ }^{29}$ Sin embargo, la prevalencia de impactos al comer varía entre $23.2 \%$ y $72.9 \%$ para niños del Reino Unido y Tailandia respectivamente. ${ }^{6}$ Las condiciones orales existentes en cada país pueden explicar, al menos en parte, las diferencias encontradas; mientras que las condiciones orales relacionadas a procesos naturales como la caída de un diente de leche, úlceras orales y la presencia de espacios debido al recambio dentario son frecuentemente reportadas en otros países, ${ }^{28}$ las condiciones relacionadas a enfermedades orales infecto-contagiosas, tales como dolor de muela y dientes sensibles así como el sangrado de encías, fueron reportadas con mayor frecuencia entre los niños entrevistados. En relación con la severidad del impacto de las condiciones orales sobre la calidad de vida $(7.77 \pm 8.64)$, nuevamente se encontraron valores bastante similares a los reportados en Francia (6.32 + $8.22 \%)$ y Tailandia $(8.85 \pm 7.4 \%)$. Aunque los estudios iniciales sobre el uso del Child-OIDP sólo presentan reportes de prevalencia y severidad, la intensidad y la extensión de los impactos ha empezado también a ser reportada. ${ }^{6}$ La idea detrás de esto es diferenciar entre por ejemplo, un niño con impactos menores (puntaje de 1) sobre seis actividades diarias y otro niño con un impacto severo (puntaje de 6) sobre sólo una actividad diaria. Aunque la severidad en ambos es idéntica (puntaje Child OIDP de 8.33); en el primer caso, el niño presenta un impacto de muy poca intensidad mientras que el segundo presenta un impacto de severa intensidad. Así mismo, la extensión del impacto permite saber cuántas de las ocho actividades diarias evaluadas estuvieron afectadas por las condiciones orales que presentaba el niño. A pesar del hecho de que los impactos sobre la calidad de vida fueron muy frecuentes entre los niños entrevistados, ellos fueron de poca o muy poca intensidad. Sólo $1.8 \%$ de los niños tuvieron impactos de severa o muy severa intensidad (Tabla 12). Debido a que algunas de las condiciones orales auto-percibidas como problemas por los escolares son eventos de muy corta duración, como la aparición de úlceras orales, la caída de un diente de leche o la sensibilidad dentaria, sus respectivas contribuciones al puntaje total son menores, lo cual se refleja en menores niveles de intensidad. Otro hallazgo interesante fue el hecho de que entre los niños con impactos, se reportó una mayor intensidad (moderada a severa) sobre sonreír y socializar que sobre el resto de actividades diarias. Aunque esto resalta la importancia que el componente social de la salud oral parece tener en la vida de los niños, en contraste con los componentes físico y psicológico, este patrón no ha sido reportado hasta la fecha. En relación con la extensión del impacto de las condiciones orales sobre la calidad de vida se encontró que muy pocos escolares reportaron impactos sobre todas las actividades diarias evaluadas $(0.5 \%)$. La mayoría de ellos reportó impactos sobre una a tres actividades diarias $(73.2 \%)$, lo cual ha sido también previamente citado por Gherunpong et al. ${ }^{6}$ Como fue previamente mencionado, las actividades diarias más frecuentemente impactadas entre los niños de la muestra fueron comer, limpiarse la boca y sonreír. Entre las condiciones orales percibidas como causas del impacto sobre la calidad de vida, los niños identificaron al dolor de muela como la principal causa para las dificultades que tuvieron durante la realización de siete de las 


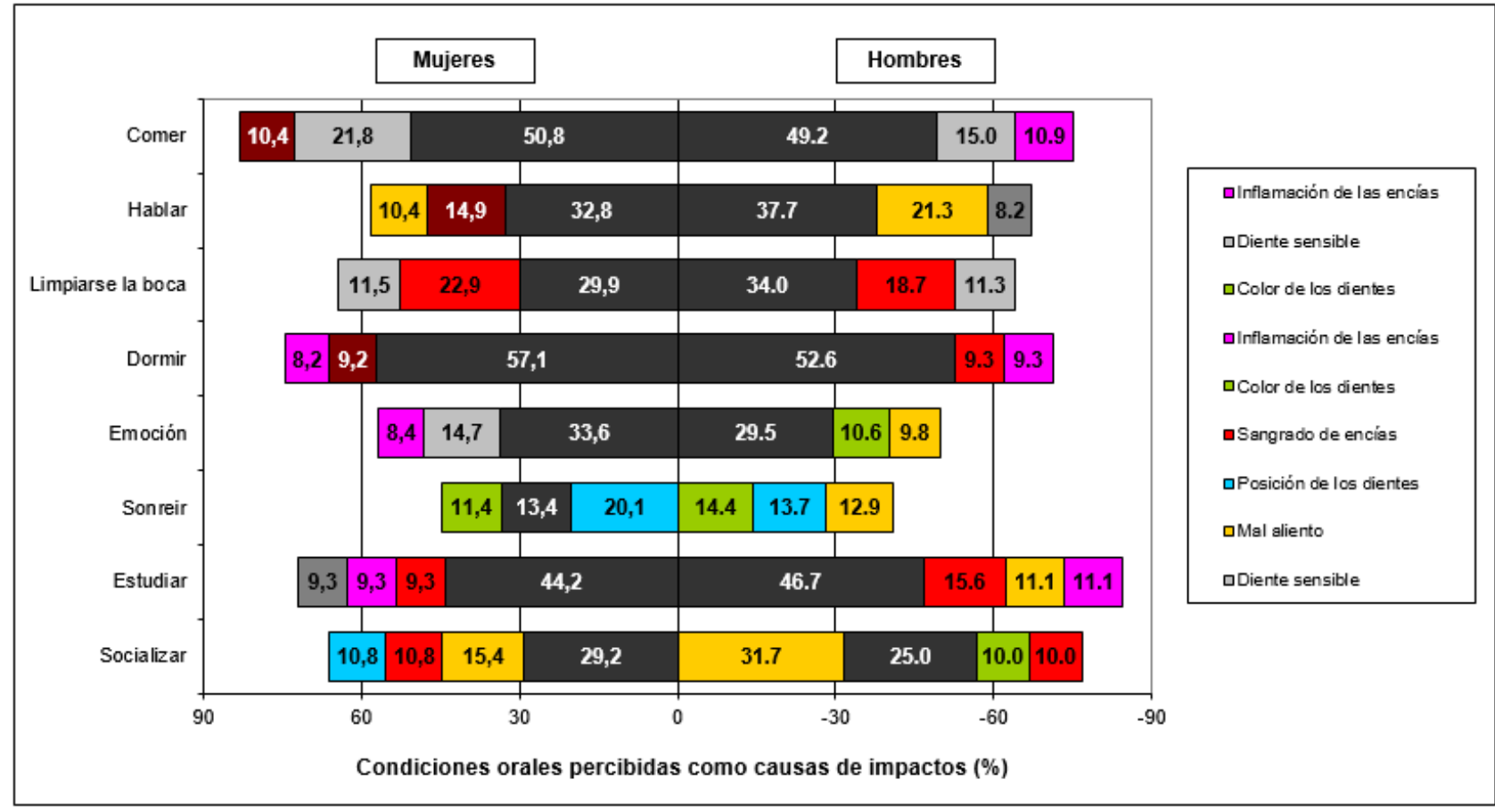

Grf. 2. Condiciones orales percibidas como causas del impacto sobre la calidad de vida en escolares de 11 y 12 años de Zapallal según sexo (Puente Piedra, 2006.)

ocho actividades evaluadas. La segunda causa más frecuente fueron el mal aliento y el sangrado de encías, que impactaron sobre cinco de las ocho actividades diarias (Gráfico 1). Estos hallazgos corroboran la idea de que entre los niños entrevistados, los síntomas asociados a las enfermedades orales más frecuentes fueron los principales causantes de impactos en la vida diaria. Aunque las alteraciones en el color y la posición de los dientes fueron menos frecuentemente reportadas por los escolares como causas de los impactos, ambas fueron las principales causas asociadas a la dificultad para sonreír, similar a lo reportado entre niños tailandeses. El impacto de las maloclusiones sobre la calidad de vida, en especial al impedir sonreír, reír o mostrar los dientes sin sentir vergüenza, ha sido previamente documentado entre adolescentes brasileños. ${ }^{19}$ Debe tenerse en cuenta también que la preocupación por la apariencia dentofacial es el principal factor para buscar tratamiento ortodóntico, inclusive por encima de los aspectos funcionales o estéticos. ${ }^{20,37}$ En el presente estudio todas las variables de interés fueron comparadas según sexo y nivel educativo; sin embargo, ninguna de ambas covariables ha sido previamente reportada en la literatura. En general se encontró que el sexo de los niños no influenciaba mayormente en la prevalencia, severidad, intensidad y extensión del impacto de las condiciones orales sobre la vida diaria de los escolares entrevistados. Por el contrario, el nivel educativo en el cual se encontraban matriculados los escolares (primaria o secundaria) si influenció las variables en estudio, encontrándose una mayor prevalencia y severidad del impacto de los problemas orales así como una mayor proporción de niños con impactos de intensidad moderada a severa junto con un mayor número de actividades diarias afectadas (extensión) en escolares de secundaria que en aquellos de primaria. Aunque el nivel educativo está estrechamente relacionado con la edad de los escolares, ésta no parece ser la única explicación para las diferencias encontradas entre niveles educativos, debido a que los promedios de edad en ambos niveles fueron bastante similares $(11.50 \pm 0.50$ años en primaria contra $11.85 \pm 0.25$ años en secundaria). Es posible que el entorno social en el cual se desenvuelve un escolar de primaria sea diferente al de uno de secundaria, lo cual se refleja en diferentes intereses y preocupaciones que afectan la vida diaria de ambos grupos. ${ }^{26}$ A nivel político, los presentes resultados refuerzan la idea de dedicar esfuerzos al desarrollo de modelos que tomen en cuenta ambos puntos de vista, necesidad normativa y percibida, para mejorar la planificación del cuidado de la salud oral y alcanzar la máxima ganancia en salud para la comunidad.

\section{CONCLUSIÓN}

Los hallazgos del presente estudio tienen implicancias prácticas y políticas. En un nivel más práctico, resaltan las limitaciones de enfocarse exclusivamente sobre necesidades 


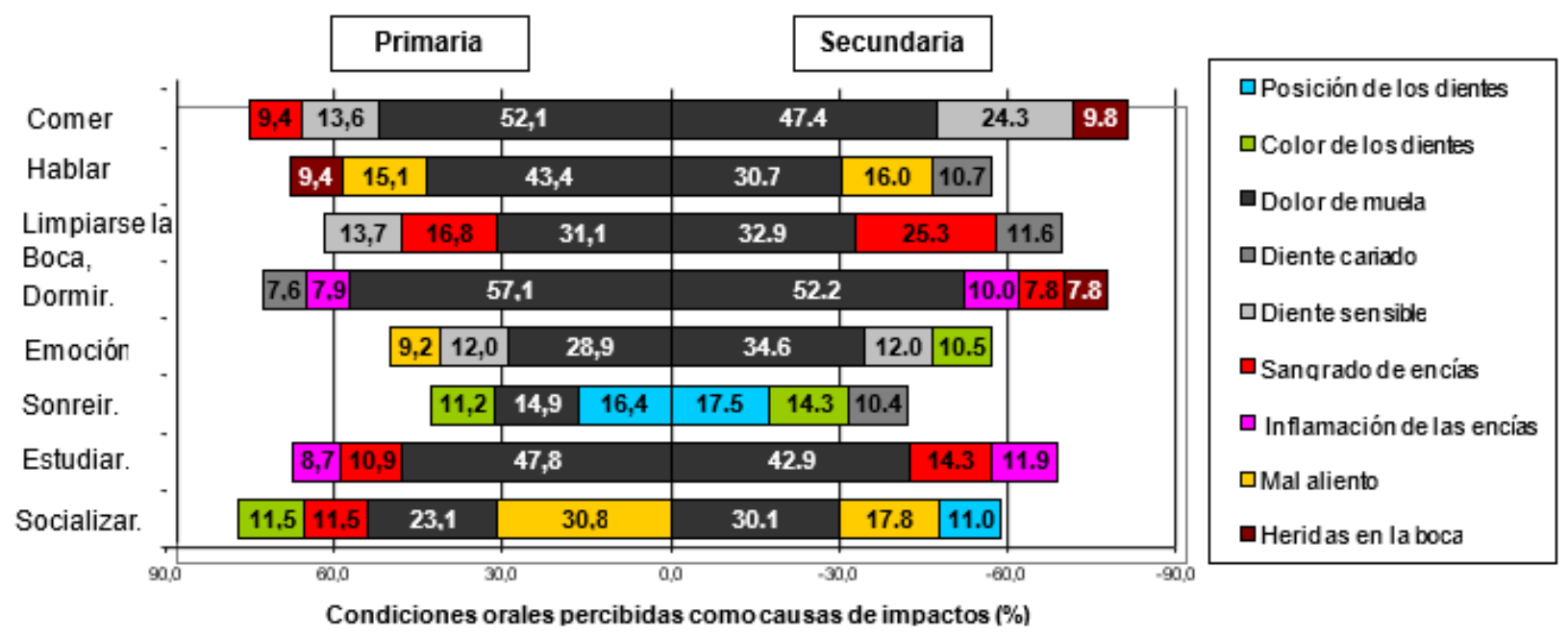

Grf. 3. Condiciones orales percibidas como causas del impacto sobre la calidad de vida en escolares de 11 y 12 años de Zapallal según nivel educativo (Puente Piedra, 2006.)

normativas, y por tanto sugieren también, la incorporación de mediciones de la calidad de vida en la evaluación de las necesidades de tratamiento, para la posterior planificación de servicios para el cuidado de la salud oral. En tal sentido, el Child-OIDP ha sido diseñado no sólo para describir el impacto de las condiciones orales sobre la calidad de vida infantil sino también para asistir en la planificación de servicios odontológicos, al complementar los indicadores clínicos durante la evaluación de las necesidades de tratamiento bucodental de una población.

Por lo tanto el presente estudio obtuvo una prevalencia muy alta del impacto de las condiciones orales sobre la calidad de vida de los niños, sin embargo la severidad no lo fue. Las dificultades para comer, limpiarse la boca y sonreír fueron los impactos más frecuentemente reportados por los niños entrevistados. Entre los niños que reportaron impactos sobre la calidad de vida, se encontró que la mayoría de los impactos fueron de poca a muy poca intensidad. Las principales condiciones orales percibidas como causas de los impactos sobre la calidad de vida estuvieron relacionadas a enfermedades orales infecto-contagiosas (dolor de muela, diente sensible y sangrado de encías).

Conflicto de intereses y financiamiento Los autores declaran no tener conflicto de intereses, haber cumplido con los requisitos de autoría y haber autofinanciado este artículo.

\section{Referencias}

1 Burt BA, Eklund SA. Dentistry, dental practice and the community. 6th ed. St. Louis: Elsevier Saunders; 2005.
2 Daly B, Watt R, Batchelor P, Treasure E. Essential dental public health. 2nd ed. New York: Oxford University Press; 2002.

3 Nutbeam D. Glossary in health promotion. Health Promotion 1986; 1: 113-127.

4 Locker D. Measuring oral health: a conceptual framework. Community Dent Health 1988; 5: 3-18.

5 Sheiham A, Steele JG, Marcenes W, Tsakos G, Finch S, Walls AW. Prevalence of impacts of dental and oral disorders and their effects on eating among older people; a national survey in Great Britain. Community Dent Oral Epidemiol 2001; 29: 195-203.

6 Gherunpong S, Tsakos G, Sheiham A. The prevalence and severity of oral impacts on daily performances in Thai primary school children. Health Qual Life Outcomes 2004; 2: 57.

7 Allen FP, Locker D. Do items weights matter? An assessment using the oral health impact profile. Community Dent Health 2004; 14: 133-138.

8 Gherunpong S, Tsakos G, Sheiham A. Developing and evaluating an oral health-related quality of life index for children; the CHILD-OIDP. Community Dent Health 2004; 21: 161-169.

9 World Health Organization. International classification of impairments, disabilities and handicaps. Geneva: World Health Organization; 1980.

10 Slade GD, Spencer AJ. Development and evaluation of the Oral Health Impact Profile. Community Dent Health 1994; 11: 3-11.

11 Slade GD. Derivation and validation of a short-form oral health impact profile. Community Dent Oral Epidemiol 1997; 25: 284-290. 
12 Adulyanon S, Sheiham A. Oral Impact on daily performan- 27 Chaves MM. Odontología Sanitaria. 2da ed. Washington: ces. In: Slade GD, editor. Measuring oral health and quality OPS: Publicaciones Científicas; 1977.

of life. Chapel Hill: University of North Carolina; 1997. p. 28 Tubert-Jeannin S, Pegon-Machat E, Gremeau-Richard C, 151-160.

Lecuyer MM, Tsakos G. Validation of a French version of

13 Adulyanon S, Vourapukjaru J, Sheiham A. Oral impacts affecting daily performance in a low dental disease Thai 29 Yusuf H, Gherunpong S, Sheiham A, Tsakos G. Validation population. Community Dent Oral Epidemiol 1996; 24: 385-389.

14 Bergner M, Bobbitt RA, Carter WB, Gilson BS. The Sickness Impact Profile: development and final revision of a 30 Bernabé E, Tsakos G, Sheiham A. Cross-cultural adaptation health status measure. Med Care 1981; 19: 787-805.

15 Robinson PG, Gibson B, Khan FA, Birnbaum W. Validity of Community Dent Health 2006; (in press). two oral health-related quality of life measures. Community 31 Tsakos G, Marcenes W, Sheiham A. Cross-cultural diffeDent Oral Epidemiol 2003; 31: 90-99.

16 Soe KK, Gelbier S, Robinson PG. Reliability and validity of two oral health related quality of life measures in Myanmar adolescents. Community Dent Health 2004; 21: 306-311.

17 Tsakos G, Marcenes W, Sheiham A. Evaluation of a modified version of the index of Oral Impacts On Daily Per- 33 formances (OIDP) in elderly populations in two European countries. Gerodontology 2001; 18: 121-130.

18 Srisilapanan P, Sheiham A. The prevalence of dental impacts on daily performances in older people in Northern 34 Thailand. Gerodontology 2001; 18: 102-108.

19 De Oliveira CM, Sheiham A. The relationship between normative orthodontic treatment need and oral health-related 35 Pan American Health Organization. Promoting Oral Health: quality of life. Community Dent Oral Epidemiol 2003; 31: 426-436.

20 de Oliveira CM, Sheiham A. Orthodontic treatment and its 36 Beltran-Aguilar ED, Estupinan-Day S, Baez R. Analysis impact on oral health-related quality of life in Brazilian adolescents. J Orthod 2004; 31: 20-27; discussion 15. of prevalence and trends of dental caries in the Americas between the 1970s and 1990s. Int Dent J 1999; 49: 322-329.

21 Srisilapanan P, Sheiham A. Assessing the difference bet- 37 Marques LS, Ramos-Jorge ML, Paiva SM, Pordeus IA. ween sociodental and normative approaches to assessing prosthetic dental treatment needs in dentate older people. Gerodontology 2001; 18: 25-34.

Malocclusion: esthetic impact and quality of life among Brazilian schoolchildren. Am J Orthod Dentofacial Orthop 2006; 129: 424-427.

22 Srisilapanan P, Korwanich N, Sheiham A. Assessing prost- 38 Gherunpong S, Tsakos G, Sheiham A. A sociodental aphodontic dental treatment needs in older adults in Thailand: normative vs. sociodental approaches. Spec Care Dentist 2003; 23: 131-134.

23 Cortes MI, Marcenes W, Sheiham A. Impact of traumatic injuries to the permanent teeth on the oral health-related quality of life in 12-14-year-old children. Community Dent Oral Epidemiol 2002; 30: 193-198. proach to assessing dental needs of children: concept and models. Int J Paediatr Dent 2006; 16: 81-88.

39 Gherunpong S, Sheiham A, Tsakos G. A sociodental approach to assessing children's oral health needs: integrating an oral health-related quality of life (OHRQoL) measure into oral health service planning. Bull World Health Organ 2006; 84: 36-42.

24 Masalu JR, Astrom AN. Applicability of an abbreviated 40 Gherunpong S, Tsakos G, Sheiham A. A socio-dental apversion of the oral impacts on daily performances (OIDP) scale for use among Tanzanian students. Community Dent proach to assessing children's orthodontic needs. Eur J Orthod 2006; 28: 393-399. Oral Epidemiol 2003; 31: 7-14.

25 Masalu JR, Astrom AN. Social and behavioral correlates of oral quality of life studied among university students in Tanzania. Acta Odontol Scand 2002; 60: 353-359.

26 Papalia DE, Wendkos S. Desarrollo físico e intelectual en la infancia intermedia. In: Papalia DE, Wendkos S, editors. Desarrollo humano. Santafé de Bogota: McGraw-Hill; 1997. p. 279-356.

Recibido: 13 de enero de 2017.

Aceptado: 17 de marzo de 2017. 